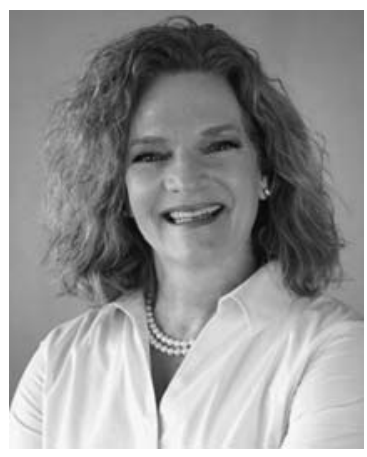

\title{
A Conversation with Elaine Mardis
}

\author{
INTERVIEWER: RICHARD SEVER \\ Assistant Director, Cold Spring Harbor Laboratory Press
}

Elaine Mardis is Robert E. and Louise F. Dunn Distinguished Professor of Medicine and co-Director, McDonnell Genome Institute at Washington University School of Medicine.

Richard Sever: I should welcome you on behalf of the journal, because you're Editor-in-Chief of Cold Spring Harbor's new journal, Molecular Case Studies. We've heard a lot about precision medicine and, in particular, " $N$ of 1 studies", which is very much a focus of the journal. Could you tell us what " $N$ of 1 studies" means in terms of precision medicine?

Dr. Mardis: Many cancer patients find themselves in a situation where they've exhausted all options they might have from FDA-approved drugs or other therapeutic interventions. They're depending on that last look at their cancer genome to reveal some new clues about treatments they might benefit from. Often, these therapies are offprotocol, or not part of a clinical trial, or even require reaching out to pharmaceutical companies to use drugs for which the tissue site of their cancer is not approved by the FDA.

When Carl June described his clinical trials with chimeric antigen receptor $\mathrm{T}$ cells, he didn't just report in terms of patient populations; he also broke down the things that he had learned from individual patients. That sort of data goes on to inform how other patients get treated in the future. Molecular Case Studies tries to make what we've learned from individual patients widely available to the whole clinical community, not just the cancer realm, so that other people can learn and maybe begin " $N$ of 2 " or " $N$ of more" studies. Individual cases, both where there have been successes but also where there have been failures to respond to a therapy that should get a response, are informative to medical care. Most of these studies involve sequencing the genome of the cancer patient tumor cells and comparing it to their normal sequence and then possibly looking at molecules like RNA or proteins for confirmation that this is really the way to go. That forms the basis for a clinical framework where we can identify the right drugs to bring the patient relief from their tumor.

Richard Sever: Someone you know was the focus of one such study. Can you tell us more about that?
Dr. Mardis: This young man, Lukas Wartman, is a colleague of mine. He's a hematologic oncologist. He wrote an important perspective for MCS to describe his experience from the standpoint of being not only a cancer doctor, but also a beneficiary of a very precise therapeutic answer that we found for him by sequencing his cancer genome. Again, this was an "off-label" (i.e., non-FDA-approved) treatment, but by virtue of our finding, he went into remission from his secondary relapse of B-cell acute lymphocytic leukemia. By virtue of being in remission, he was able to receive an unrelated stem cell transplant that saved his life. He's alive and well today. That was in $2011-5$ years out from his cancer. He's obviously committed to research, but he's also actively treating patients in the clinic and studying more about heme malignancies. He's even spoken on a personal level as a sort of advocate to patients who've been diagnosed.

Richard Sever: You've also been working on personalized cancer vaccines as immunotherapy against melanoma. How does that work?

Dr. Mardis: Much like an invading bacterium or virus, the proteins that are produced by cancer cells often look different than normal proteins in normal cells. Cancer mutations at the level of the DNA mean changes in the amino acid sequences in the proteins. Those modified peptides, or "neoantigens," that are unique to the cancer cells should look different to the body's defense system. We know that cancers find ways to suppress the immune cells around them so they go undetected, so these mutant proteins go unattacked by immune molecules that would normally target the cell for death.

The vaccine approach uses genomics to tell us which amino acids are changed and of those, which ones are likely to elicit a strong immune response from vaccination. Those sequences are identified by comparing to the nonmutated sequences from the patient in the context of their specific immune molecule sequences

(C) 2016 Mardis. This article is distributed under the terms of the Creative Commons Attribution-NonCommercial License, which permits reuse and redistribution, except for commercial purposes, provided that the original author and source are credited. 
(HLA). The proteins that bind most avidly to the HLA should be the ones that elicit the strongest immune response. The vaccine approach is meant to re-educate the patient's immune system about what it should recognize and to overcome that immune suppression by stimulating it to produce specific $\mathrm{T}$ cells that will now identify those tumor-specific proteins. We get a lot of other information out of the genomics component, including identifying specific human leukocyte antigen molecules. Those are the molecules that will identify and bind the mutant proteins. More than anything, it's a computational prediction of those peptides that are most non-self.

Richard Sever: Has it been challenging to develop the ability to do that?

Dr. Mardis: It has, and we haven't solved it completely. At this point, there are more questions than answers. That's not a reason to not try these modalities, especially in patients that have exhausted their possibilities. We're pretty good at choosing neoantigens, but not as good as we need to be.

Richard Sever: How many potential targets do you start with that you have to refine down to a manageable number for use in a vaccine?

Dr. Mardis: Typically, we choose the top ten peptides. That's an arbitrary number because we don't know the right number. Another area of interest is that so far we've chosen single point mutations that result in a single amino acid substitution. That peptide looks different, but imagine how much more different it would look if, instead of a single point mutation, we've shifted to a different reading frame in the DNA. You would likely add a completely unique peptide sequence to its end that isn't present in the normal protein. Another example might be if two genes in the cancer DNA end up fused together: BCR-Abl and the Philadelphia chromosome are nice examples of that. Such modifications could be unique to a patient. They might not be in drivers like the BCR-Abl, but they could make a completely unique peptide that would then elicit a strong immune response.

Richard Sever: Unlike an oncogene driver, it doesn't matter what the peptide does; it's just the fact that it's different. It's a flag saying, "I'm a tumor cell. Come get me."

Dr. Mardis: Exactly. Of course, if any of these neoantigens were also parts of known driver genes, we would definitely target them because you could shut down the cancer cell on multiple levels. Most neoantigens, however, turn out to be passenger mutations. Usually when we try to marry specific targeted therapies to specific mutations, we really go after the known driver genes and tend to think of these passenger mutations as rather mundane. In the context of vaccine therapy, they turn out to be incredibly valuable. It's a different philosophical approach to the genomic data.
Richard Sever: You're validating this with RNA-seq. Can you explain what that means and why?

Dr. Mardis: On a practical level, we don't want to put a peptide in the vaccine that is not actually being produced by the cancer cell. In an ideal world, we would choose our peptides and look for them in protein data, say, from mass spectrometry. Currently, it's not practical to go that far because mass spectrometry is less sensitive than DNA sequencing and requires more tissue that we often have available. Instead, we look at the RNA expression of specific mutations that correspond to the neoantigens we want in our vaccine. This is especially important in high mutation load tumors like melanomas. We have lots of neoantigens to choose from, but by looking carefully at the RNA-seq data, we know that only $\sim 50 \%-60 \%$ of the predicted neoantigens are actually produced as RNA. It's a pretty good intermediate as to whether a gene is actually being expressed and, in particular, whether that altered gene is being expressed in the tumor cells.

Richard Sever: How do you make the vaccine once you've got the peptide sequence?

Dr. Mardis: So far, we've tried synthesizing the peptides in a high-quality GMP [good manufacturing practices]compliant way and then marrying them with specifically cultured dendritic cells from that patient. The dendritic cells are the parts of the immune system that present these peptides to the major histocompatibility complex and direct the $\mathrm{T}$ cells to be produced against the peptides. You basically co-opt the delivery vehicle from that patient, marry it up with your neoantigen peptides, and then infuse it back into the patient as a vaccine. That's one way. In a simpler method, we just produce a peptide or set of peptides that correspond to the neoantigens, dissolve them in an adjuvant much like you would a normal vaccine, and inject intramuscularly. They find their own way and marry up with the various immune molecules, and off the immune system goes.

Richard Sever: You're counting more on the body there than doing it ex vivo. Presumably, that would be more scalable?

Dr. Mardis: Presumably. Although the peptides can be quite expensive, there are commercial entities that are working on rapid GMP-compliant synthesis of all the peptides for each patient together. They just set aside a specific room for that patient's peptide collection of neoantigens, and the turnaround time is about a week. This is a good way of condensing down what can be a rate-limiting step. The dendritic cell approach takes longer because they first have to be isolated from the patient, specifically cultured, married up with the peptide cocktail, and then returned to the patient over a schedule of infusions. It's a bit more involved.

We can also make RNA and package it according to the neoantigens, or we can work completely back upstream and insert DNA corresponding to the different peptides that we want to produce into a vector just behind a human 
promoter. These get electroporated into the patient's cells and injected back into the patient, so the cells themselves make the neoantigens. There are many forms and flavors to this, all of which are being worked on by a variety of groups. Each approach has its own merits, its own associated costs, and its own associated difficulties or challenges to produce and to scale. If this is ever going to catch on as a way to battle cancer, it has to happen in a clinically relevant time frame. In other words, can we do all of this in a time frame and at a cost that meets the patient's needs in terms of where they're at in their disease fight?

Richard Sever: You're looking at melanoma now. How far can one go with this?

Dr. Mardis: We're focusing on the high-mutation-load cancers because they have more neoantigens to choose from. We're focusing on a second trial in melanoma, and we have a trial open in triple-negative breast cancer. We're also looking at relapse gliomas in children and glioblastomas in adults. Many of these patients after their first diagnoses and removal of their tumor receive a specific chemotherapy called temozolomide that has been shown in $\sim 30 \%$ of patients to actually increase the mutation rate significantly when their cancer recurs. This is a special situation that we can take advantage of, because now these patients have the mutation load that gives us lots of things to choose from in our search for neoantigens. We also want to work toward understanding tumors with a lower mutation rate. In essence, how low can we go in terms of the number of neoantigens that are chosen but still have an effective vaccine? That's an important and completely unanswered question. 


\section{$\$_{\text {CSH\& }}^{\infty}$ Cold Spring Harbor Symposia SYMPOSIA}

\section{A Conversation with Elaine Mardis}

Cold Spring Harb Symp Quant Biol 2016 81: 328-330 originally published online January 19, 2017

Access the most recent version at doi:10.1101/sqb.2016.81.031633
Creative This article is distributed under the terms of the
Commons http://creativecommons.org/licenses/by-nc/4.0/, which permits reuse and
License redistribution, except for commercial purposes, provided that the original author and source are credited.

Email Alerting Receive free email alerts when new articles cite this article - sign up in Service the box at the top right corner of the article or click here. 